\title{
I JORNADA DE LA CELEBRACIÓN DEL DÍA INTERNACIONAL DEL COMBATE A LA CORRUPCIÓN
}

SUMARIO; Introducción II.Inauguración. III. Intervención Doctora Fabiola Luna Navarro.IV.Participación Maestro Jaime Hernández Colorado

\section{RESUMEN}

En el documento se da cuenta de lo acontecido durante la IJornada de la celebración del día internacional del combate a la corrupción, en la que participaron la Doctora Fabiola Navarro Luna, del Instituto de Investigaciones Jurídicas de la UNAM, IIJUNAM, quien disertó magistralmente sobre "El Papel de la academia y la ciudadanía", seguida de la intervención del Maestro Jaime Hernández Colorado ${ }^{1}$ denominada "El municipio como reto del Sistema Nacional Anticorrupción: diagnóstico y buenas prácticas"; concluyendo, tan importante evento, con la presentación de la conferencia magistral del Doctor Jorge Fernández Ruiz de la Universidad Nacional Autónoma de México UNAM "El combate a la corrupción en México".

PALABRAS CLAVE: Academia, combate a la corrupción,municipios

\section{DAY OF THE CELEBRATION OF INTERNATIONAL DAY OF THE FIGHT AGAINST CORRUPTION}

\begin{abstract}
The document realizes what happened during the I day of the celebration of the international day of the fight against corruption, in which Dr. Fabiola Navarro Luna, from the Institute of Legal Investigations of the UNAM, IIJUNAM, who spoke masterfully on "The Role of academia and citizenship" followed by the intervention of Master Jaime Hernández Colorado called "The municipality as a challenge of the National Anti-Corruption System: diagnosis and good practices"; concluding, so important event, with the presentation of the keynote lecture of Dr. Jorge Fernández Ruiz of the National Autonomous University of Mexico UNAM "The fight against corruption in Mexico."
\end{abstract}

KEY WORDS: Academy, fight against corruption, municipalities

\section{RESEÑA}

\footnotetext{
${ }^{1}$ Investigador,Coordinador Ejecutivo del Programa Interdisciplinario de Rendición de Cuentas del Centro de Investigación y Docencia Económicas CIDE de la Comunidad PIRC-RRC CIDE,e-mail: jaime.hernandez@cide.edu
} 
El pasado mes de diciembre de 2019, la Red del Programa Interdisciplinario de Rendición de Cuentas (PIRC), del Centro de Investigación y Docencia Económicas (CIDE), donde participan maestros e investigadores de las Divisiones de Ciencias Sociales y de Ciencias Económicas y Administrativas de la Universidad de Sonora, realizó, bajo la temática: "Sistema Nacional Anticorrupción: Tareas pendientes desde sus inicios y a 12 meses de la 4T" ( teniendo como sede el auditorio Astolfo Chavarín, edificio 9Q2, planta baja de la División de Ciencias Económicas y Administrativas de la Universidad de Sonora) la I Jornada de la Celebración del Día Internacional del Combate a la corrupción." La inauguración de tan relevante evento estuvo a cargo de la directora de la División de Ciencias económico administrativas, Doctora María Elena Robles Baldenegro, quién enfatizó Que la rendición de cuentas es una práctica que fortalece las instituciones democráticas y el Estado de derecho, y que las naciones que obligan a sus funcionarios públicos y gubernamentales a informar de manera periódica e institucionalizada,suelen ser las que ostentan mayores condiciones de vida, economías más fuertes y ejercen una mejor defensa de las libertades individuales.

"Si un Estado no garantiza la rendición de cuentas, y los ciudadanos no tienen acceso a una información fidedigna y adecuada de las acciones de gobierno, entonces existirán mayores riesgos de que las instituciones públicas se corrompan",

En otra parte de su intervención, la doctora Robles Baldenegro hizo referencia al Convenio que tiene la Universidad de Sonora con el Centro de Investigaciones y Docencia Eonómicas CIDE, al que se le reconoce por su gran avance en el tema de la Rendición de Cuentas, institución con la cual, ya se han iniciado los trabajos conjuntos en una amplia gama de temas relacionados con el combate a la corrupción y tópicos asociados, enfatizando además la importancia de que los académicos e investigadores, establezcan vínculos con los gobiernos, empresas e instituciones, para trabajar y aportar en conjunto, soluciones que disminuyan el flagelo de la corrupción así como el índice de percepción al 
respecto.Siguiendo con el orden de las intervenciones de los reconocidos expertos en el tema, procede mencionar : en primer término,la ilustrativa intervención de la Doctora Fabiola Navarro Luna, del Instituto de Investigaciones Jurídicas de la UNAM, IIJUNAM, quien disertó magistralmente sobre "El Papel de la academia y la ciudadanía", seguida de la intervención magistral del Maestro Jaime Hernández Colorado" denominada "El municipio como reto del Sistema Nacional Anticorrupción: diagnóstico y buenas prácticas"; para cerrar, tan importante evento, con la presentación de la conferencia magistral del Doctor Jorge Fernández Ruiz de la Universidad Nacional Autónoma de México UNAM "El combate a la corrupción en México".

Una vez expuestos, los puntos de vista de los conferencistas invitados, en la sesión de preguntas y respuestas, intervinieron con interesantes cuestionamientos[integrantes de la sociedad civil, y del Sistema Estatal Anticorrupción,autoridades locales y municipales de control y fiscalización,expresidentes y extesoreros municipales,representantes de órganos no gubernamentales, estudiantes de los posgrados de administración pública, fiscalización y control gubernamental, entre otros asistentes] a los cuales, dieron amplia y convincentes respuestas los expositores. Con ello se ratificó, lo subrayado por el doctor Aaron Grajeda Bustamante, en entrevista, previa a la celebración de la precitada Jornada Académica ${ }^{3}$,

“...sobre la importancia de la vinculación entre academia, sociedad civil y gobierno, como una de las potencialidades para coadyuvar, particularmente, con la masa crítica y el acopio de ideas a través de la inteligencia colectiva para buscar innovar, dentro de este tipo de iniciativas ciudadanas, en la reducción de la corrupción en nuestro estado..."

${ }^{2}$ Investigador,Coordinador Ejecutivo del Programa Interdisciplinario de Rendición de Cuentas del Centro de Investigación y Docencia Económicas CIDE de la Comunidad PIRC-RRC CIDE,e-mail: jaime.hernandez@cide.edu

3 Noticias UNISON,Se realizará en la Unison la Primera Jornada del Día Internacional contra la Corrupción,Luis Mendoza,7 de diciembre de 2019[en línea] https://www.unison.mx/nota/?idnoti=29299; consultado el 10 de diciembre de 2019 
I Jornada de la celebración del día Internacional del combate a la corrupción Año 11, Número 21 julio-diciembre de 2019 Jaime Hernández Colorado

A continuación la Síntesis de la intervención del Maestro Jaime Hernández Colorado "El municipio como reto del Sistema Nacional Anticorrupción: diagnóstico y buenas prácticas". El tema lo dividió en III Secciones, la primera denominada: I.Municipio y Sistemas Anticorrupción, en la que magistralmente presentó una panorámica de las etapas acontecidas en el entorno local, federal y municipal desde las Reformas Constitucionales que dieron vida al actual Sistema Nacional Anticorrupción y a las de sus homólogos en las entidades federativas; asi mismo, el conferencista hernández planteó que si no se incluyen los gobiernos municipales en este tema, se corre el riesgo elevado de que todas estas estrategias de combate a la corrupción y de rendición de cuantas fracasen, sencillamente porque los municipios actualmente son el orden de gobierno más importante que existe en el país, hasta nuestros dias, y por ello en los que es necesario, accionar

\section{Municipio y Sistemas Anticorrupción}

- Las reformas constitucionales y legales que han vertebrado a la iniciativa mexicana para el combate a la corrupción, estrategia que se cristaliza en los sistemas anticorrupción, han tenido la obligación de traducirse en legislación estatal. Estas regulaciones locales diseñaron roles diferentes para los municipios.

- En sentido general se evidencia un vacío; la ausencia de un proyecto claro para incluir a la institución municipal en el combate a la corrupción. Es posible que esa falta de líneas de acción se vincule con que el orden de gobierno municipal en México ha sido dejado de lado tanto por políticas públicas de auge reciente, como por la discusión pública acerca de las reformas necesarias para la mejora del ejercicio gubernativo en el país.

- En el diseño legal anticorrupción de las entidades federativas se evidencia el problema grave de que la estrategia no necesariamente ha incluido a los municipios.

- Ese olvido normativo puede generar no sólo confusión, sino espacios para el incumplimiento o la simulación por parte de los entes municipales, como sujetos obligados. Por otro lado, la exclusión de los municipios tiene capacidad para transformarse en un problema de tal calado que puede hacer naufragar los esfuerzos anticorrupción.

- El análisis de las leyes estatales permite trazar un listado de problemas derivados de la heterogeneidad con que se ha tratado a los municipios. En diecinueve de las leyes estatales anticorrupción de todo el país se 
establece que los municipios son integrantes de los sistemas estatales y/o de su comité coordinador.

De esos estados:

- Chiapas, Coahuila, Colima, Guerrero, Nayarit, Sinaloa, Tlaxcala y Zacatecas apuntan genéricamente que los municipios concurrirán a los sistemas estatales, a través de sus representantes;

- Aguascalientes, Durango, Guanajuato, Hidalgo, Michoacán y Morelos señalan que los municipios participarán por medio de las contralorías;

De esos estados

- Baja California establece la participación de los municipios mediante los síndicos procuradores;

- Sonora y Chihuahua cuentan con una redacción muy similar, en la que se establece que los municipios participarán en los términos que señale el Comité Coordinador, sin embargo, la Constitución del Estado de Chihuahua se refiere a la figura del síndico —como medio para la participación municipal—;

- La Constitución del Estado de México sostiene que la participación municipal se hará por vía de los Sistemas Municipales Anticorrupción, quienes concurrirán a través de sus presidentes rotatoriamente, conforme a los dieciocho distritos judiciales en que se divide el territorio de la entidad;

- La legislación de la Ciudad de México establece que los titulares de las Alcaldías serán invitados permanentes y participarán en las sesiones del Comité Coordinador, aunque sólo con derecho a voz.

Del análisis de las diecinueve legislaciones que prevén la participación de los municipios en los sistemas locales anticorrupción, se puede extraer una suerte de tipología de los mecanismos de dicha participación. Como es claro:

- En algunos casos, la participación municipal se establece mediante los representantes de las áreas de control interno (contraloría y/o sindicatura).

- En otros casos, la participación municipal se establece mediante mecanismos de representación indirecta, sin permitir que concurran todos los municipios.

- En algunos ejemplos, la participación se limita otorgando voz, pero no voto a los gobiernos municipales en los comités coordinadores.

- Finalmente, en otros diseños se establece la participación municipal, a reserva de que la regule una norma diferente o que los términos de ésta los diseñen los comités coordinadores. 
I Jornada de la celebración del día Internacional del combate a la corrupción Año 11, Número 21 julio-diciembre de 2019

Jaime Hernández Colorado

En las diecinueve leyes estatales señaladas, se habla indistintamente de los municipios como parte de los sistemas anticorrupción, pero únicamente en cinco de ellas se considera la representación municipal en los comités coordinadores de dichos sistemas.

Es decir, de los 19 estados que incluyen a los municipios, sólo 5 establecen su participación en las instancias rectoras de combate a la corrupción. Y uno más, la Ciudad de México, hace parte del comité coordinador a los titulares de las alcaldías, aunque con carácter de "invitados permanentes", sólo con derecho a voz.

En la II Sección Combate a la corrupción municipal, de la magistral disertación del Maestro Jaime Hernández Colorado, se mencionaron las acciones sustantivas que se propone al efecto, así como los objetivos que con ellas se pretende alcanzar y la metodología a seguir: El maestro Hernández además de mencionar el vacío y falta de proyecto para los municipios, en el escenario del combate a la corrupción, afirmó, que es probable que ello está vinculado con el rol marginal que se ha dado a los municipios, además de una circunstancia de desventaja respecto a los otros dos órdenes de gobierno. Situación que a su vez, ha terminado por marginarlos y arrebatarles las atribuciones que tienen en términos de diseño constitucional. Y que por lo tanto, ello, ha derivado en dos circunstancias que no son menores: por una parte, la del incumplimiento de las obligaciones legales, y la otra, la del cumplimiento simulado. Seguidamente el expositor señalo que los municipios cuentan con los recursos necesarios para atender esa temática, además de que tienen la posibilidad de acompañarse en su función cotidiana de la observación y vigilancia de los ciudadanos. “

II. Combate a la corrupción municipal

- Las acciones sustantivas que se proponen se enfocan a:

- Aclarar los mandatos y los procesos asociados a éstos.

- Limitar la discrecionalidad de los funcionarios públicos.

- Anclar el modelo de combate a la corrupción en la rendición de cuentas

- Desarrollar herramientas que introduzcan el principio de la responsabilidad activa en el servicio público

Ejes de la propuesta de Política Nacional Anticorrupción de la Comunidad PIRCRRC. 
- Adaptación al ámbito municipal.

\begin{tabular}{l|l} 
POLÍTICA NACIONAL & \\
ANTICORRUPCIÓN & ACCIONES SUSTANTIVAS
\end{tabular}

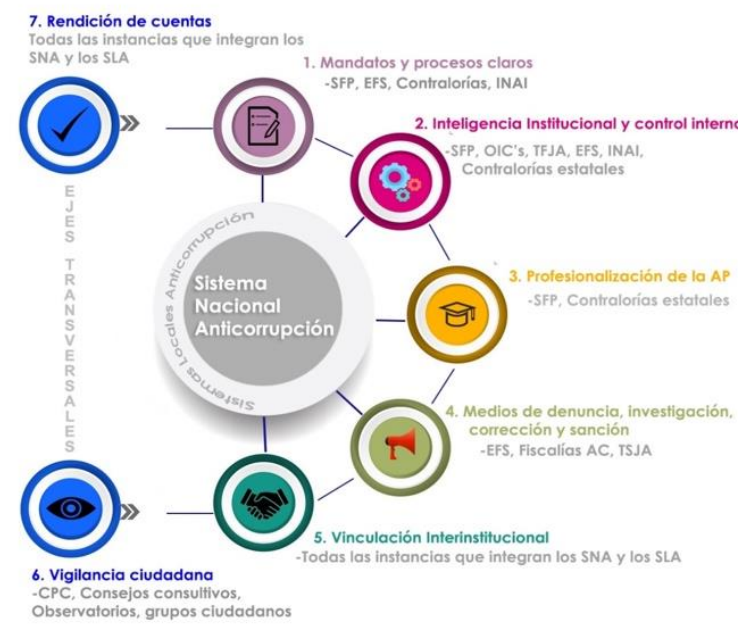

- Las acciones sustantivas que se proponen como ejes esenciales están diseñadas para desdoblarse en acciones de política en los ámbitos estatal y municipal. En esos niveles de gobierno deben iniciar por el establecimiento de un sistema de rendición de cuentas, con la finalidad de garantizar que existan las condiciones institucionales indispensables para la puesta en marcha de esas acciones sustantivas.

Acción Inicial. Rendición de cuentas para el combate a la corrupción en estados y municipios.

- Objetivo: Instrumentar un sistema de rendición de cuentas en los gobiernos estatales y municipales, que se articule como la condición base en la cual se cimentarán las acciones estatales $-y$ municipales-derivadas de la política nacional anticorrupción.

- Etapa 1. Transparencia legal como base mínima para la rendición de cuentas y el combate a la corrupción.

- Etapa 2. Mejora de la gestión y buenas prácticas en todas las vertientes de la rendición de cuentas.

- Etapa 3. Política de transparencia proactiva.

- Etapa 4. Procesos administrativos simplificados para la transparencia y la rendición de cuentas permanentes. 
I Jornada de la celebración del día Internacional del combate a la corrupción

- Los municipios mexicanos tienen el imperativo de responder desde el ámbito administrativo a todas las obligaciones que les han sido asignadas en años recientes, y en el caso concreto del combate a la corrupción, la atribución de imponer sanciones.

- Si existe claridad acerca del imperativo de cumplir y de todas las reglas que deben respetarse, es evidente que los municipios mexicanos se enfrentan a varios retos, entre ellos:

- Identificar todas las leyes, reglamentos y, en general, el marco normativo completo que le asigna obligaciones que deben cumplirse.

- Reconocer y clasificar todas las obligaciones que asigna el marco normativo, lo que implica distribuirlas en las diferentes dependencias que integran las administraciones municipales para distribuir también las responsabilidades de cumplimiento.

- Introducir en las administraciones una nueva lógica de gestión, un nuevo comportamiento burocrático que permita responder a los desafíos que significan las exigencias normativas que tienen los gobiernos municipales.

- La simplificación administrativa tiene que ver con la nueva lógica de gestión, no sólo se hace referencia a la urgencia de clarificar los procesos mediante los cuales se atiende a la ciudadanía, sino también a aclarar aquellos que no tienen salida directa a ella, pero que sí inciden en la eficacia y eficiencia de las administraciones.

- Culminación de los otros cuatro retos: la formación de cuerpos profesionales que: 1) conozcan los marcos legales aplicables, 2) conozcan las obligaciones que les atañen, por área de la administración, 3) respondan a una nueva lógica de gestión que les permita cumplir las obligaciones municipales de diversa índole, y, 4) conozcan, instrumenten y mejoren los procedimientos administrativos con el objetivo de optimizar los recursos de la administración en el funcionamiento interno de ésta.

Asimismo, el expositor mencionó los

Tres imperativos que forman parte de la esencia de las leyes:

- Transparencia - Entendida como el cumplimiento de las obligaciones legales, de principio pero también como una estrategia que permite cumplir con las exigencias de las leyes en la materia y, además desarrollar acciones proactivas que permiten ir más allá de esas exigencias a fin de garantizar de que la ciudadanía se pueda enterar. 
- Rendición de cuentas - Entendida como el ejercicio permanente de informar y justificar las decisiones públicas frente a la ciudadanía e instituciones contrapeso.

- Combate a la corrupción - Entendiendo a esta como la apropiación ilegítima de lo público. Tiene que ver con el abuso $Y$ continuar apuntando de la autoridad para obtener beneficios privados.

Las acciones iniciales que se deben emprender para hacer frente a los cinco retos municipales, respetando los imperativos que se han descrito, son:

1. Establecer perfiles de puesto, acordes con las atribuciones de cada área de la administración.

2. Articular un sistema de gestión de archivos municipales, de acuerdo con los instrumentos de la archivística que se establecen en la legislación de transparencia y archivos.

3. Desarrollo de una plataforma de transparencia propia, acorde a las posibilidades de los gobiernos municipales, que permita consultar la información de obligaciones de transparencia en tiempo real.

4. Introducir buenas prácticas de contabilidad, manejo presupuestal y adquisiciones y contrataciones públicas.

5. Diseño de nuevos procedimientos administrativos municipales que modifiquen y, sobre todo, simplifiquen los procesos que ha de seguir cada dependencia municipal, tanto en el ámbito interno, como en aquellos espacios de relación con la ciudadanía.

6. Diseño de herramientas sencillas, de principio, de transparencia proactiva, que acerquen la información pública a la ciudadanía.

En la III Sección denominda Acciones concretas, en cuanto a la aclaración de mandatos, profesionalización y obligaciones de transparencia para los municipios, el Maestro Jaime Hernández Colorado mencionó las siguientes

III. Acciones concretas

1) Aclaración de mandatos:

a) Identificación de atribuciones de cada área de la administración pública municipal;

b) Identificación de atribuciones de cada funcionario, según espacio de la administración municipal en que se desempeñe;

c) Identificación de procedimientos administrativos que son trámites y materializan las atribuciones:

a) Pasos, características del proceso, responsables, producto, duración, costo (si aplica);

b) Elaboración de diseños del proceso y flujos. 
I Jornada de la celebración del día Internacional del combate a la corrupción

c) Publicación de procesos y flujos (en internet e in situ).

2) Elementos básicos de profesionalización:

a) Identificación del total de puestos de la administración pública municipal.

b) Con base en atribuciones, diseño de perfiles de puesto.

c) Criterios básicos de reclutamiento, selección y contratación basados en perfiles de puesto.

d) Capacitación mínima de los funcionarios, en tres materias:

a) Obligaciones de transparencia (qué corresponde a quién);

b) Procedimientos administrativos que le corresponden a cada funcionario, según sus atribuciones;

c) Atención al público.

3) Obligaciones de transparencia:

a) División de obligaciones por área, conforme a atribuciones.

b) Establecimiento de un procedimiento general de cumplimiento, conforme a términos de la ley para cada tipo de información.

c) Parámetro de cumplimiento, según momento de generación de la información.

d) Responsables de acopio final de la información y actualización de la página.

De igual manera,para cerrar la Sección III de su intervención, en relación al punto 4) Herramientas de gobierno abierto,el maesto Hernández Colorado, expuso detalladamente, con la proyección en pantalla, algunos ejemplos, de la forma de transparentar de diversos Estados y municipios del país, sus actuaciones ante la ciudadanía, utilizando para ello sus Portales digitales de Transparencia, a saber:

4) Herramientas de gobierno abierto:

a) En el municipio de Querétaro la Consulta vía internet de la ejecución de obras públicas y sesión de adquisiciones

Asimismo:

b)Ejemplificó la forma en que en el Estado de Veracruz presenta a los ciudadanos en su Portal de Transparencia y Acceso a la información, la Sección Consulta de Obras y Acciones por Municipio, dando a conocer además a los contribuyentes del impuesto predial, la forma en las que ellos pueden utilizar la aplicación para verificar el procedimiento conforme al cual, excepcionalmente las autoridades hacendarias les determina las cuotas a pagar por cada inmueble.

c) Igualmente,mostró la forma de transparentar el municipio de Veracruz los Estados financieros, acorde a lo dispuesto en el Manual de Contabilidad Gubernamental publicado en el Diario Oficial de la Federación del 6 de Octubre de 2014.

y finalmente

d)Presentó la página de Portal de Transparencia del Estado de Missouri (MAP)Missouri Accountability Portal donde se puede consultar la actividad 
gubernamental, la ejecución de obras públicas y demas actividades en las que se ejerce el presupuesto de egresos, el cual es un modelo a seguir por los municipios,entidades federativas y ámbito de gobierno federal en tratandose de la accountability como la forma óptima de trabajar en una organización con Responsabilidad, proactividad y compromiso.

Por su parte, la Doctora Fabiola Luna Navarro, Académica y Coordinadora del Observatorio contra la Corrupción y la Impunidad OCl, del Instituto de Investigaciones Jurídicas de la UNAM disertó sobre "El papel de la academia y la ciudadanía, mencionando en primer término, la bondad de las tecnologias de la información y de la comunicación para generar nuevo conocimiento en relación a todo aquello relacionado con posibles actos de corrupción y su posible detección temprana, evitando así el desvío de recursos millonarios de los gobiernos.

De igual manera, la doctora Luna Navarro, mencionó la importancia de la colaboración académica como una perspectiva de estudio distinta y complementaria a lo normativo o conductual de las personas que participan en actos de corrupción... con el objetivo de identificar cuáles son los mecanismos y patrones que hacen posible que sucedan tales actos, y en contrapartida... tomar medidas para prevenirlos y evitar que vuelvan a suceder".

En su intervención, mencionó, que el Observatorio contra la Corrupción y la Impunidad $\mathrm{OCI}$, con sede en el Instituto de Investigaciones Jurídicas y la Facultad de Ciencias Políticas y Sociales de la UNAM, tiene como objetivo de estudio totalmente académico, generar y difundir conocimiento en el tema de la corrupción e impunidad en México. Así, con la participación de académicos y estudiantes ---se monitorean--- las denuncias sobre conductas de corrupción y se identifican comportamientos de quienes intervienen en ellas. Ademas se estudia el entorno en el que se inserta el Sistema Nacional Anticorrupción en México, desde el enfoque de los actores y sus resultados.Y también se examinan aquellos factores que puedan impactar en la efectividad de las políticas de combate a la corrupción

Dado que el Observatorio contra la Corrupción y la Impunidad $\mathrm{OCI}$, tiene como objetivo producir conocimiento de tipo académico, desde la universidad, se generan lineas de investigación, de las cuales surgen diversos proyectos cuyos hallazgos sobre la corrupción, además de explicar el fenómeno que tanto preocupa 


\section{Jornada de la celebración del día Internacional del combate a la corrupción}

y perjudica a la sociedad en general,son susceptibles de pubicación en revistas científica. De otra parte, la doctora Luna Navarro puntualizó que "Las universidades tienen que tener el compromiso de impulsar y desarrollar la ciencia aplicada y que el conocimiento que se genera, también pueda coadyuvar a cambiar lo que se sabe no funciona como debiera.

Igualmente la conferencista mencionó que si bien a nivel mundial no han encontrado que se esté realizando trabajo similar o con igual enfoque, lo que si se conoce que se utiliza, son las ciencias de la complejidad y la teoría de redes, sobre todo para el estudio de la "delincuencia organizada o el narcotráfico"

Finalmente la doctora Fabiola Luna Navarro concluyó, mencionando que en relación al tema de la corrupción, se han vinculado con otras universidades de nuestro país y del extramjero y que el próximo año estarán haciendo nuevos trabajos colaborativos en este tema. 